\title{
Regular Exercise, Quality of Life, and Mobility in Parkinson's Disease: A Longitudinal Analysis of National Parkinson Foundation Quality Improvement Initiative Data
}

Miriam R. Rafferty ${ }^{\mathrm{a}, *}$, Peter N. Schmidt ${ }^{\mathrm{b}}$, Sheng T. Luo ${ }^{\mathrm{c}}, \mathrm{Kan} \mathrm{Li}^{\mathrm{d}}$, Connie Marras $^{\mathrm{e}}$, Thomas L. Davis ${ }^{\mathrm{f}}$, Mark Guttman ${ }^{\mathrm{g}}$, Fernando Cubillos ${ }^{\mathrm{b}}$, Tanya Simuni ${ }^{\mathrm{h}}$ and on behalf of all NPF-QII Investigators ${ }^{\mathrm{i}}$

${ }^{a}$ Center for Education in Health Sciences, Northwestern University, Chicago, IL, USA

${ }^{\mathrm{b}}$ National Parkinson Foundation, Miami, FL, USA

${ }^{\mathrm{c}}$ Department of Biostatistics, University of Texas Health Science Center at Houston, Houston, TX, USA

${ }^{\mathrm{d}}$ Department of Biostatistics, University of Texas Health Science Center at Houston, Houston, TX, USA

${ }^{\mathrm{e}}$ Morton and Gloria Shulman Movement Disorders Centre and the Edmond J Safra Program in Parkinson's disease, Toronto Western Hospital, University of Toronto, Toronto, Canada

${ }^{\mathrm{f}}$ Thomas L. Davis, Division of Movement Disorders, Department of Neurology, Vanderbilt University, Nashville, TN, USA

${ }^{\mathrm{g}}$ Division of Neurology, Department of Medicine, University of Toronto, Toronto, Canada

${ }^{\mathrm{h}}$ Department of Neurology, Northwestern University, Chicago, IL, USA

${ }^{\mathrm{i}}$ All National Parkinson Foundation - Quality Improvement Initiative (NPF-QII) Centers

and site principal investigators are listed in the Appendix

Accepted 24 October 2016

Abstract.

Background: Research-based exercise interventions improve health-related quality of life (HRQL) and mobility in people with Parkinson's disease (PD).

Objective: To examine whether exercise habits were associated with changes in HRQL and mobility over two years.

Methods: We identified a cohort of National Parkinson Foundation Quality Improvement Initiative (NPF-QII) participants with three visits. HRQL and mobility were measured with the Parkinson's Disease Questionnaire (PDQ-39) and Timed Up and Go (TUG). We compared self-reported regular exercisers $(\geq 2.5$ hours/week) with people who did not exercise 2.5 hours/week. Then we quantified changes in HRQL and mobility associated with 30-minute increases in exercise, across PD severity, using mixed effects regression models.

Results: Participants with three observational study visits $(n=3408)$ were younger, with milder PD, than participants with fewer visits. After 2 years, consistent exercisers and people who started to exercise regularly after their baseline visit

\footnotetext{
${ }^{*}$ Correspondence to: Miriam R. Rafferty, Center for Education in Health Sciences, Northwestern University, 633N. St Clair 20th
} 
had smaller declines in HRQL and mobility than non-exercisers $(p<0.05)$. Non-exercisers worsened by 1.37 points on the PDQ-39 and a 0.47 seconds on the TUG per year. Increasing exercise by 30 minutes/week was associated with slower declines in HRQL ( -0.16 points) and mobility $(-0.04 \mathrm{sec})$. The benefit of exercise on HRQL was greater in advanced PD ( -0.41 points) than mild PD $(-0.14$ points; $p<0.02)$.

Conclusions: Consistently exercising and starting regular exercise after baseline were associated with small but significant positive effects on HRQL and mobility changes over two years. The greater association of exercise with HRQL in advanced PD supports improving encouragement and facilitation of exercise in advanced PD.

Keywords: Parkinson disease, exercise, quality of life, mobility limitations, rehabilitation

\section{INTRODUCTION}

Parkinson's disease (PD) is associated with progressive mobility-related impairments that predict decreased health-related quality of life (HRQL) [1] and increased mortality [2]. One of the earliest signs of impaired mobility is decreased walking activity, which can occur prior to measurable declines in gait speed or balance [3]. Increasing walking, general physical activity, and regular exercise are lifestyle factors that may be associated with better HRQL and mobility over time in PD [4].

Previously completed studies demonstrate that a variety of exercise modalities are associated with improved HRQL and functional mobility in people with PD. Examples of these formal, supervised exercise interventions include progressive resistance exercise [5, 6], treadmill training [7], Tai Chi [8], Tango [9], and PD-specific exercise programs [10, 11]. However, few of these clinical trials deliver exercise interventions for greater than 6 months $[5,6$, 11]. It is currently unknown whether people with PD who change or maintain regular exercise behavior, outside of supervised research-based exercise interventions, have better HRQL and mobility outcomes over time.

An initial cross-sectional analysis from the National Parkinson Foundation Quality Improvement Initiative (NPF-QII) clinical study revealed that selfreported regular exercise was associated with better mobility, HRQL, and reduced caregiver strain [12]. In that study, participation in exercise was categorized as none, low (less than 2.5 hours per week), or high (greater or equal to 2.5 hours per week). Greater or equal to 2.5 hours per week of exercise is significant because the American Academy of Neurology has recently updated their quality metrics for people with PD to include yearly counseling on the importance of regular exercise, defined as at least 2.5 hours per week of moderate intensity activity per week [13]. The early analysis of the NPF-QII study indicated that baseline exercise was a significant predictor of HRQL, mobility, physical function, caregiver strain, and disease progression at the one year follow up visit $(\mathrm{N}=2252)$ [12].

As more data have been collected in the NPFQII study, longitudinal analysis provides valuable information about how exercise relates to long-term outcomes. The purpose of this analysis is to examine the extent to which regular exercise impacts HRQL and functional mobility over 2 years. The primary objective of this analysis was to identify whether maintaining regular exercise, or improving exercise habits to greater or equal to 2.5 hours/week, shown to be beneficial at baseline [12], was associated with sustained benefits in HRQL and functional mobility over 2 years. The other objectives were to quantify the impact of increasing exercise by a 30-minute increment per week, and to determine whether the impact of exercise differed across PD severity.

\section{METHODS}

The NPF-QII is an international, multicenter, prospective clinical study of care and outcomes that has recorded data from 21 sites in North America, the Netherlands, and Israel identified as Centers of Excellence (Centers) by the National Parkinson Foundation. Information on the development of the NPF-QII research study has been published previously [15]. Participants were eligible for the study if they received medical care for the diagnosis of PD at one of the participating centers, with no other exclusions. Participants were included in this analysis if they had data collected during at least 3 annual visits. The Institutional Review Boards at each Center approved the data collections, and informed consent was obtained from each participant. De-identified data collected from August 20, 2009 to September 28,2015 was used for this analysis. 
The NPF-QII study collects demographics, disease duration, Hoehn and Yahr stage (HY), family history, brief neuropsychological profile and cognitive assessments, as well as data on pharmacologic and non-pharmacologic management of PD symptoms [15]. These observational study visits are scheduled on a yearly basis. At each visit, exercise is measured by the self-reported number of hours per week of exercise. Additional information regarding the type and intensity of exercise is not available in this dataset. The Parkinson Disease Questionnaire (PDQ39) measured patient-reported, PD-specific HRQL $[16,17]$. Functional mobility was measured by the Timed Up and Go (TUG) test, in which performance is tested by timing participants as they rise from a chair, walk 3 meters, turn, and return to sitting in the chair [18]. Higher values of the PDQ39 indicate worse HRQL and higher TUG times indicate slower performance, or worse functional mobility.

\section{Participants}

In the first key analysis, participants who exercised greater or equal to 2.5 hours per week were classified as regular exercisers (E), and were hypothesized to experience health benefits compared to non-exercisers and low-exercisers [12]. The non- and low-exercisers (identified as $\mathrm{N}$ ) were expected to have less benefit due to participation in less than 2.5 hours of exercise per week. Over time, as people increased or decreased their exercise over 2 years, or 3 visits, we identified 8 groups: NNN, NNE, NEE, EEE, ENN, EEN, ENE, NEN. Decreasing or stopping exercise could be related to a choice to reduce exercise due to change in motivation or available time, but could also be due to a functional decline or illness that was not related to exercise habits. To avoid confounding our interpretation of exercise habits with potential illnesses we focused our first key analysis only on groups NNN, NNE, NEE, and EEE, which are defined in Table 1. Data for all 8 groups are reported in Supplementary Table 1.

The second key analysis included all participants with at least 3 recorded visits. Including all participants allowed the analysis to account for both increases and decreases in time spent exercising.

\section{Statistical analyses}

First, the longitudinal (3 visit) cohort of the NPFQII participants was compared to the cohort who had less than 3 visits (two sample $t$-test, Wilcoxon Rank Sum, Fisher's exact test, and Pearsons chisquare test as appropriate). Then in the first key analysis, we compared our four groups of interest: consistent regular exercisers, those starting to exercise after the first or second visit, and consistent non-exercisers. We present (1) absolute HRQL and functional mobility, (2) risk-adjusted HRQL and functional mobility values, and (3) risk-adjusted change from baseline. The risk adjustment includes the following baseline variables: age at diagnosis, disease duration, sex, disease severity, and comorbidities. Comorbidities were categorized as 0,1 , or $2+$ based on the participant's response to having one of the following conditions: heart problems, respiratory problems, diabetes, cancer, arthritis, other neurologic disorder, and other non-neurologic disorder. Because age and disease duration had a non-linear relationship with HRQL and functional mobility, they were treated by coding each as splines with 2 interior knots $(0.3,0.6)$. Pairwise comparisons of mean change from baseline at year two (3rd visit) were performed using a 2 sided $t$-test with the presented $p$-values adjusted for multiple comparisons using the Holm method.

Our second key research question used multivariate, mixed effects, linear regression to quantify the changes in HRQL and functional mobility associated with increases and decreases in exercise over time. Zero exercise was the reference point, and

Table 1

Group definitions

\begin{tabular}{lll}
\hline Abbreviation & Title & Definition \\
\hline NNN & Consistent non/low-exercisers & Exercise $<2.5$ hours/week at all 3 visits \\
NNE & Late-starting exercisers & Began exercising $\geq 2.5$ hours/week after 2nd study visit \\
NEE & Early-starting exercisers & Began exercising $\geq 2.5$ hours/week after 1st study visit \\
EEE & Consistent exercisers & Exercise $\geq 2.5$ hours/week at all 3 visits \\
\hline ENN & Preliminary analyses revealed that participants who decreased their exercise activity had \\
EEN & corresponding declines in mobility and quality of life. Analysis of these groups require \\
ENE & further exploration & \\
NEN & & \\
\hline
\end{tabular}


the upper limit was 15 hours of exercise per week. Exercise was recorded in hours per week using decimals for time less than an hour. For the purpose of this analysis, exercise was rescaled as a continuous variable with increasing units of 30 -minutes $(0.5 \mathrm{hrs})$, as 30 minutes is a clinically-relevant increment of exercise. Time spent exercising was treated as a time-varying covariate to account for increases or decreases in exercise over time. The time variable was measured in years from first visit to account for inter-individual variability in visit scheduling. Two random effects were included in the models: intercept to account for baseline inter-individual differences, and linear slope of time to account for inter-individual differences in progression. The same risk adjustment methods used in the first analysis were used (age at diagnosis, disease duration, sex, disease severity, and comorbidities). A secondary analysis compared the results between disease severity groups rather than controlling for disease severity. This secondary analysis tested whether the impact of exercise differed in people with moderate (HY stage 3) and advanced PD (HY stage 4-5), compared to the reference group of mild PD symptoms (HY stage 1-2).

\section{RESULTS}

\section{Participants}

The current analyses included 3408 participants who had data over 2 years, representing at least 3 clinic visits. The second visit occurred on average at $1.28 \pm 0.49$ years after the first visit and the third visit occurred on average at $2.49 \pm 0.67$ years after the first visit. Demographic and baseline disease characteristics of the participants are presented in Table 2. Our cohort represents $42 \%$ of the total 8041 NPFQII participants. Reasons for exclusion from this analysis included the following: approximately $12 \%$ of participants had not reached 2 years following enrollment; $7 \%$ died prior to their third visit; $14 \%$ withdrew from the study (moved away from Center, changed site of PD care, PD is no longer the diagnosis, or declined further participation in study). At the time of this analysis, an additional $25 \%$ of the original participants had not achieved 3 visits for unknown reasons. The resulting analyses include 3,189 participants who completed the PDQ-39 and 3,062 participants who completed the TUG. More participants in the advanced PD (HY 4-5) category

Table 2

Baseline characteristics of NPF-QII participants, comparing those with $\geq 3$ Visits and those with $<3$ visits

\begin{tabular}{|c|c|c|c|c|}
\hline & $\begin{array}{c}\text { All NPF-QII } \\
\text { Participants }\end{array}$ & $\begin{array}{c}\text { Participants } \\
\text { with } \geq 3 \text { Visits* }\end{array}$ & $\begin{array}{c}\text { Participants } \\
\text { with }<3 \text { visits }\end{array}$ & $\begin{array}{c}\text { Difference } \\
\text { between groups } \\
\text { s }\end{array}$ \\
\hline $\mathrm{N}(\%)$ & 8041 & $3408(42.40 \%)$ & $4633(57.60 \%)$ & \\
\hline $\mathrm{N}$ Male $(\%)$ & $5061(62.94 \%)$ & $2153(63.17 \%)$ & $2908(62.77 \%)$ & $0.7^{\mathrm{a}}$ \\
\hline Age at Start of Study & $67.15 \pm 9.91$ & $65.92 \pm 9.36$ & $68.06 \pm 10.19$ & $<0.0001$ \\
\hline Time since PD diagnosis & $7.157 \pm 5.87$ & $7.154 \pm 5.64$ & $7.16 \pm 6.03$ & 0.13 \\
\hline HY & $2.34 \pm 0.80$ & $2.26 \pm 0.74$ & $2.41 \pm 0.84$ & $<0.0001$ \\
\hline HY 1-2 (n) & 4575 & 2118 & 2457 & \\
\hline HY 3 (n) & 2263 & 940 & 1323 & $<0.0001$ \\
\hline HY 4-5 (n) & 596 & 163 & 433 & \\
\hline Exercise All (hrs/week) & $3.02 \pm 4.53$ & $3.16 \pm 5.15$ & $2.92 \pm 4.01$ & 0.02 \\
\hline HY 1-2 $(n=5169)$ & $3.46 \pm 5.05$ & $3.53 \pm 5.78$ & $3.41 \pm 4.33$ & 0.45 \\
\hline HY $3(n=2863)$ & $2.60 \pm 3.75$ & $2.78 \pm 3.88$ & $2.47 \pm 3.66$ & 0.05 \\
\hline HY 4-5 $(n=1199)$ & $1.64 \pm 2.49$ & $1.58 \pm 2.04$ & $1.66 \pm 2.63$ & 0.68 \\
\hline PDQ-39 Total Score All & $0.25 \pm 0.16$ & $0.22 \pm 0.15$ & $0.26 \pm 0.16$ & $<0.0001$ \\
\hline HY $1-2(n=5182)$ & $0.20 \pm 0.13$ & $0.19 \pm 0.13$ & $0.20 \pm 0.14$ & 0.001 \\
\hline HY $3(n=2870)$ & $0.31 \pm 0.15$ & $0.29 \pm 0.15$ & $0.33 \pm 0.16$ & $<0.0001$ \\
\hline HY 4-5 $(n=1203)$ & $0.43 \pm 0.15$ & $0.41 \pm 0.14$ & $0.43 \pm 0.15$ & 0.15 \\
\hline TUG All (sec) & $10.94 \pm 3.62$ & $10.44 \pm 3.24$ & $11.36 \pm 3.86$ & $<0.0001$ \\
\hline HY $1-2(n=4444)$ & $10.40 \pm 3.13$ & $9.99 \pm 2.82$ & $10.75 \pm 3.34$ & $<0.0001$ \\
\hline HY $3(n=1768)$ & $12.26 \pm 4.18$ & $11.52 \pm 3.78$ & $12.87 \pm 4.39$ & $<0.0001$ \\
\hline HY 4-5 $(n=502)$ & $16.33 \pm 5.60$ & $16.26 \pm 6.45$ & $16.36 \pm 5.23$ & 0.66 \\
\hline
\end{tabular}

Except for subject counts and sex, data shown are mean \pm standard deviation. *Participants with $\geq 3$ annual visits (baseline, follow up year 1, and follow up year 2) were included in analyses. ${ }^{\dagger}$ Participants with $<3$ visits recorded were excluded from analysis. ${ }^{\S} p$-value for comparison between the $\geq 3$ visit (included) group and the $<3$ visit (excluded) group were completed using two sample $t$-tests, Wilcoxon Rank Sum (time since diagnosis), Fisher's exact test (sex), or Pearson's Chi-square (HY). $P$-values for exercise, PDQ-39, and TUG were adjusted for multiple comparisons. 
were missing TUG scores. The baseline demographics and measures of interest for the cohort of included participants $(n=3,408)$ are compared to the excluded cohort $(n=4,633)$ in Table 2 . The participants who were included in the analysis, having at least 3 visits, tended to be younger, with earlier stage PD, higher levels of exercise, better HRQL and functional mobility compared to the participants with less than 3 visits.

\section{Effects of exercising or starting to exercise at least 2.5 hours per week}

The absolute values of HRQL and functional mobility for the 4 exercise groups are shown in Fig. 1A and 1B. The consistent non-exercisers have worse HRQL and worse functional mobility than each of the other three groups at each visit. The risk-adjusted $\mathrm{HRQL}$ and functional mobility measures presented in Fig. 1C and 1D demonstrate that controlling for age, disease duration, sex, disease severity, and comorbidities does not account for differences between non-exercisers and exercisers at baseline, although the three groups that began as nonexercisers had similar functional mobility at baseline (Fig. 1D). Because of initial differences between groups, we completed our statistical analysis on the risk-adjusted change from baseline (Fig. 1E and 1F). Maintaining regular exercise at least 2.5 hour/week (EEE) and becoming a regular exerciser after the baseline visit (NEE) were associated with better HRQL and functional mobility after 2 years compared to consistent non-exercisers. Participants who became regular exercisers later, after their second

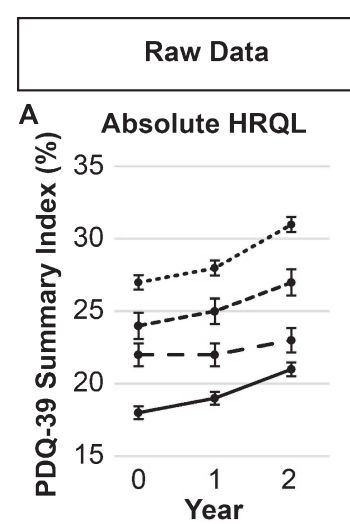

B Absolute Mobility

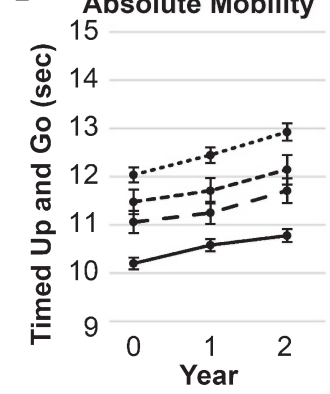

\section{Risk-Adjusted Data}

C Risk-Adjusted HRQL

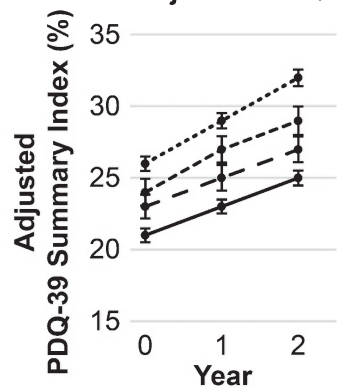

D Risk-Adjusted Mobility

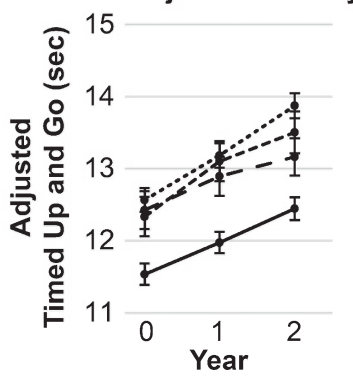

Risk-Adjusted
Change from Baseline

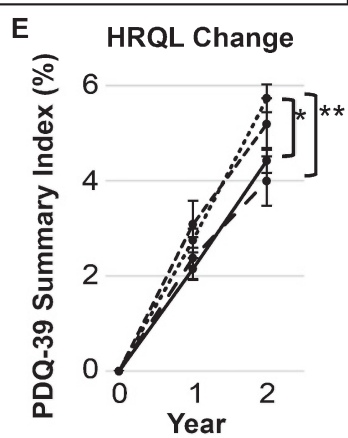

F Mobility Change

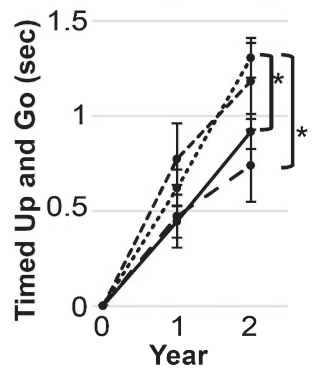

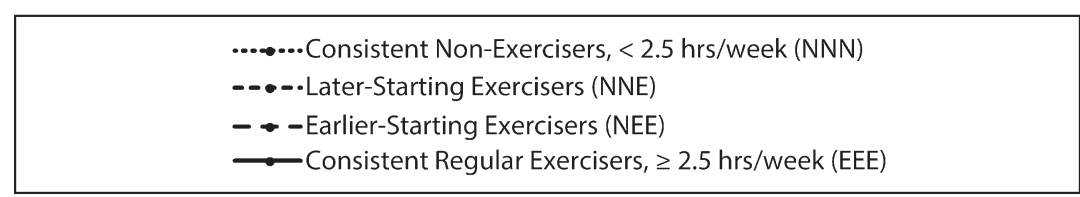

Fig. 1. Health-related quality of life and mobility over two years. Absolute (A and B), risk-adjusted (C and D), and risk-adjusted change from baseline (E and F) health-related quality of life (A, C, and E) and mobility (B, D, and F) by exercise classification across three visits, or approximately 2 years. Risk-adjusted values in C-F are controlled for age, sex, disease duration, disease severity, and comorbidities. Analyses are performed only on risk-adjusted change from baseline (E-F) due to non-equivalence at baseline for absolute and risk-adjusted HRQL and mobility (A-D). *indicates Holm-adjusted $p<0.05 .{ }^{* *}$ indicates Holm-adjusted $p<0.01$. Abbreviations: HRQL, Health-related quality of life; hrs, hours; PDQ-39, Parkinson's disease questionnaire. 
visit (NNE), were not different from the consistent non-exercisers.

\section{Quantifying changes associated with 30-minute exercise increments over time}

Controlling for age, sex, disease duration, disease severity, and comorbidities, non-exercisers worsened by 1.37 points (SE 0.08 ) per year in the PDQ-39, and worsened by 0.47 seconds (SE 0.03 ) in their TUG time. Across all participants, a 30-minute increase in exercise per week was associated with 0.16 point (SE 0.02 ) better PDQ-39 score per year and by $0.04 \mathrm{sec}-$ onds (SE 0.01) better TUG performance per year $(p<0.0001$ for all). The secondary analysis presented in Table 3 compares participants with moderate (HY 2.5 - 3) and advanced PD (HY 4-5) to the reference group of mild PD (HY 1-2). This analysis revealed that people with advanced $P D$ had the largest improvements in HRQL related to exercise $(-0.41$ points inclusive of reference group value, compared to -0.14 points in mild PD; $p=0.02$ ). For functional mobility, the improvements were similar at all PD stages.

\section{DISCUSSION}

The NPF-QII study is the largest prospective, longitudinal, observational study of people with PD followed in the naturalistic setting. This is the first analysis reporting longitudinal outcomes of exercise habits over two years. The first key finding of this analysis was that self-reported regular exercise habits at least 2.5 hours per week, was associated with significantly less decline in HRQL and functional mobility over two years compared to people who did not exercise regularly. Furthermore, participants who started to achieve greater or equal to 2.5 hours per week after their first study visit also demonstrated significantly better HRQL and functional mobility after two years compared to those who remained non- or low-exercisers. These findings suggest that regular exercise, even when started later in the course of PD, may slow the decline in HRQL and functional mobility over the long-term in people with PD. However, beginning regular exercise after the $2 \mathrm{nd}$ visit, did not achieve statistically significant protection on HRQL or functional mobility when measured approximately one year later. Thus, the benefits of regular, but informal, independent exercise habits may require a longer time to accrue than short-term, supervised, researchbased exercise participation [5-11].

The small, but statistically significant, benefit of regular exercise habits on HRQL and functional mobility was measured as a slower decline in PDQ-39 and TUG performance. In contrast, exercise trials can result in improvements in HRQL and mobility in 6 weeks to 6 months [5, 7-9]. The exercise performed in clinical trials is performed with greater supervision, structure, intensity, and external motivation than self-reported exercise in the NPF-QII data [5-11], which is likely to contribute to the greater gains. Exercise trials frequently include 2-3 hours/week $[5,6,8,9]$. Some exercise trials only include participants with low exercise behaviors prior to the study to maximize measureable improvements $[5,6]$. Although we cannot assume a causal relationship, our results suggest that there are long-term benefits to sustained regular exercise habits, even if it is not in a formal, protocol-driven, supervised research

Table 3

Effect of increasing exercise by 30 minutes on HRQL and mobility in people with mild $\mathrm{PD}$, compared to moderate and advanced PD

\begin{tabular}{|c|c|c|c|}
\hline & \multicolumn{3}{|c|}{ Reference Group } \\
\hline & $\begin{array}{l}\text { Mild PD } \\
\text { HY 1-2 }\end{array}$ & $\begin{array}{c}\text { Moderate PD } \\
\text { HY } 3\end{array}$ & $\begin{array}{c}\text { Advanced PD } \\
\text { HY 4-5 }\end{array}$ \\
\hline Quality of Life $(n=3189)$ & $(n=2048)$ & $(n=896)$ & $(n=245)$ \\
\hline PDQ-39 Total (\%) & $\beta(\mathrm{SE})$ & $\beta(\mathrm{SE})$ & $\beta(\mathrm{SE})$ \\
\hline Time & $1.37(0.08)^{*}$ & & \\
\hline Exercise & $-0.14(0.02)^{*}$ & $-0.04(0.04)$ & $-0.27(0.12)^{* *}$ \\
\hline Mobility ( $n=3036$ ) & $(n=2138)$ & $(n=822)$ & $(n=102)$ \\
\hline \multicolumn{4}{|l|}{ TUG (Sec) } \\
\hline Time & $0.47(0.03)^{*}$ & & \\
\hline Exercise & $-0.04(0.01)^{*}$ & $-0.01(0.02)$ & $0.01(0.05)$ \\
\hline
\end{tabular}

Data presented are parameter estimate $(\beta)$ followed by the standard error of the regression coefficient, controlled for age, sex, disease duration, and comorbidities. *Indicates significance $(p<0.0001)$ in mild PD. ${ }^{* *}$ Indicates significant comparison to reference group $(p<0.05)$. 
setting. In this observational study, the exercise selfreported by the participants could include a variety of exercise modalities and intensities over the course of a week, as long as the participant defined it as exercise time. Our observation of exercise benefits, despite the self-reported and general nature of the exercise recorded, supports the American Academy of Neurology's recommendation of regular exercise counseling for people with PD [13].

The incremental benefit of increasing exercise by 30 minutes per week was also small but significant: less than 1 point on the PDQ-39 and less than $0.1 \mathrm{sec}-$ onds for the TUG per year. A 1.6 point change on the PDQ-39 over 6 months has been shown to be the smallest clinically important difference in people with PD [19] and the standard error of measurement of PDQ-39 is approximately 4 points [20]. Although there is no established clinically important difference for the TUG in people with PD, the minimal detectable change $(95 \%$ confidence interval) values range from 3.5 to 11 seconds [21-23]. Based on these estimates of clinical responsiveness, the benefits of adding 30 minutes regular exercise per week would not be measurable on an individual level after only one year. Increasing exercise by more than 30 minutes, or maintaining that increase in exercise for greater than 2 years, are likely to be necessary to measure individual differences over time. The first analysis suggests that the risk-adjusted change in PDQ-39 was approximately 1.6 points different between the consistent exercisers and non-exercisers (Fig. 1C), suggesting that 2.5 hours of exercise per week over 2 years, rather than a 30 minute difference over 1 year, reaches clinically meaningful thresholds. Future experimental studies will need to establish clinically meaningful frequency, intensity, and duration of informal exercise. The regression analysis may be interpreted more clearly as the better natural history of exercisers compared to the non-exercising reference group.

An unexpected finding was that the HRQL benefit associated with 30-minute increases in exercise per week was greatest in people with advanced PD. While the incremental difference was small this finding has significant clinical and research implications for the development of strategies to make exercise and physical activity more accessible to people with more severe disability. People with more advanced PD may have poor access to regular exercise, as their mobility impairments would limit their independent participation in existing community and group exercise programs. Community-based exercise programs may also increase caregiver burden in people with advanced PD, because they may rely on their caregiver for transportation [24]. In addition, most exercise intervention studies [5-9] and studies presenting community-based exercise programs $[10,11]$ target people with mild to moderate PD symptoms. Thus, novel methods to encourage physical activity and exercise in people with advanced PD, particularly methods that account for caregiver concerns, could provide a great benefit to the PD community. The smaller improvements seen in HRQL in people with mild PD may be due to lower level of impairment and a possible floor effect on the PDQ-39 [17]. However, our average impairment of $0.19 \pm 0.13$ on the PDQ-39 for people with mild PD (Table 2) indicates that many of the participants still had some room for improvement.

In contrast to HRQL, the association of exercise with functional mobility was similar across all stages of PD. Interpretation of consistency across all stages could be limited in people with advanced PD, as people who could not complete the TUG without using their hands were not included in this analysis. Using an alternate TUG analysis, the "TUG with penalty" [25], that adds 6 seconds for the use of hands to push up from the chair would include more participants, but would be more difficult to interpret as its psychometric properties have not yet been established. As clinicians and researchers investigate the effect of exercise in future studies with more advanced PD participants, developing clinical responsiveness data for the "TUG with penalty" may improve the interpretation of this measure.

One limitation that impacts both analyses is that the included participants with at least 3 visits represented only $42 \%$ of the entire NPF-QII study. These participants were younger, more likely to be in the early stages of PD during the first visit, they had better HRQL and functional mobility at baseline, and exercised more than the whole NPF-QII sample. Thus, these results may not generalize to all people with PD nor to people who are not receiving treatment for their PD at expert care sites or movement disorders neurology clinics. Future longitudinal analyses will benefit from longer duration of follow up, inclusion of more participants, and advanced statistical techniques including imputation of missing data. These longer duration studies may be able to measure changes in the trajectory of HRQL and mobility when participants start exercising using nonlinear regression analyses. A second limitation is that we cannot assume a causal relationship between 
exercise and better HRQL and functional mobility over 2 years based on the results of this observational study. A third limitation is that the NPF-QII data does not include type or intensity of exercise performed. Since this data was analyzed, intensity of exercise was added to the NPF-QII data collection form, which will provide more clinically relevant information about how to prescribe regular exercise in future analyses. A fourth limitation is that the self-reported exercise measure could be prone to inaccuracies and reporting bias. There have been great advances in body-worn sensors to measure exercise since the NPF-QII study was initiated in 2009. Sensors are likely to have better reliability and responsiveness than self-reported data, but questions remain about the most sensitive devices and analysis algorithms to define activity across the stages of the disease [26]. In the absence of body-worn sensors, the substantial baseline differences between exercisers and non-exercisers suggest that participants provide acceptable estimations of their activity and ability [27, 28]. However, the baseline differences remaining between exercisers and non-exercisers after risk-adjustment indicates that exercisers and non-exercisers have other fundamental differences that are not explained by age, disease duration, disease severity, sex, and number of comorbidities. Some motor symptoms of PD are roughly accounted for in our analysis by the disease severity metric. Other unmeasured differences between exercisers and non-exercisers may include non-motor symptoms of Parkinson's disease and patient-centric factors, such as depression, apathy, and self-efficacy.

In conclusion, beginning to exercise regularly after baseline, or reporting consistent exercise over 2 years were both associated with small but significant protective effects on HRQL and functional mobility over two years. The greater benefit of exercise on HRQL in advanced PD suggests that clinicians should encourage, facilitate, and monitor long-term exercise participation across all stages of PD. Although these observational data only scratch the surface of what could be measured for exercise, HRQL, mobility, and potential confounding variables, these data are a valuable addition to existing research on regular exercise habits in people with PD.

\section{ACKNOWLEDGMENTS}

This research was funded by the National Parkinson Foundation. Miriam Rafferty also received funding support from Northwestern University's
Integrated Post-doctoral Fellowship in Health Services and Outcomes Research (Administration for Community Living, National Institute of Disability, Independent Living, and Rehabilitation Research grant H133P130013; PI: Allen Heinemann, PhD). Sheng Luo's research was also supported in part by the National Institute of Neurological Disorders and Stroke under Award Number R01NS091307 and by the National Center for Advancing Translational Sciences under Award Number KL2-TR000370. These contents do not necessarily represent the policy or endorsement of the funders. The authors would like to thank all the NPF-QII investigators, Center coordinators, and participants without whom this work would not be possible. NPF-QII Centers and Site PIs are listed in the Appendix.

\section{CONFLICT OF INTEREST DISCLOSURE FOR PRESENT STUDY}

All authors receive salary, research support, and/or honoraria from the National Parkinson Foundation.

\section{SUPPLEMENTARY MATERIAL}

The adjusted means of the TUG and PDQ-39 data for all 8 groups (NNN, NNE, NEE, EEE, ENN, EEN, ENE, NEN) are included in Supplementary Table 1.

The supplementary material is available in the electronic version of this article: http://dx.doi.org/ 10.3233/JPD-160912.

\section{REFERENCES}

[1] Stewart KC, Fernandez HH, Okun MS, Jacobson CE, \& Hass CJ (2008) Distribution of motor impairment influences quality of life in Parkinson's disease. Mov Disord, 23, 14661468.

[2] Lo RY, Tanner CM, Albers KB, Leimpeter AD, Fross RD, Bernstein AL, McGuire V, Quesenberry CP, Nelson LM, \& Van Den Eeden SK (2009) Clinical features in early Parkinson disease and survival. Arch Neurol, 66, 1353-1358.

[3] Cavanaugh JT, Ellis TD, Earhart GM, Ford MP, Foreman KB, \& Dibble LE (2012) Capturing ambulatory activity decline in Parkinson's disease. J Neurol Phys Ther, 36, 51-57.

[4] Speelman AD, van de Warrenburg BP, van Nimwegen $M$, Petzinger GM, Munneke M, \& Bloem BR (2011) How might physical activity benefit patients with Parkinson disease? Nat Rev Neurol, 7, 528-534.

[5] Corcos DM, Robichaud JA, David FJ, Leurgans SE, Vaillancourt DE, Poon C, Rafferty MR, Kohrt WM, \& Comella CL (2013) A two-year randomized controlled trial of progressive resistance exercise for Parkinson's disease. Mov Disord, 28, 1230-1240. 
[6] Prodoehl J, Rafferty MR, David FJ, Poon C, Vaillancourt DE, Comella CL, Leurgans SE, Kohrt WM, Corcos DM, \& Robichaud JA (2015) Two-year exercise program improves physical function in Parkinson's disease: The PRET-PD randomized clinical trial. Neurorehabil Neural Repair, 29, 112-122.

[7] Harro CC, Shoemaker MJ, Frey O, Gamble AC, Harring KB, Karl KL, McDonald JD, Murray CJ, VanDyke JM, Tomassi EM, \& VanHaitsma RJ (2014) The effects of speeddependent treadmill training and rhythmic auditory-cued overground walking on balance function, fall incidence, and quality of life in individuals with idiopathic Parkinson's disease: A randomized controlled trial. Neurorehabilitation, 34, 541-556.

[8] Li F, Harmer P, Liu Y, Eckstrom E, Fitzgerald K, Stock R, \& Chou LS (2014) A randomized controlled trial of patientreported outcomes with tai chi exercise in Parkinson's disease. Mov Disord, 29, 539-545.

[9] Hackney ME, \& Earhart GM (2009) Health-related quality of life and alternative forms of exercise in Parkinson disease. Parkinsonism Relat Disord, 15, 644-648.

[10] Cugusi L, Solla P, Zedda F, Loi M, Serpe R, Cannas A, Marrosu F, \& Mercuro G (2014) Effects of an adapted physical activity program on motor and non-motor functions and quality of life in patients with Parkinson's disease. Neurorehabilitation, 35, 789-794.

[11] Steffen T, Petersen C, \& Dvorak L (2012) Communitybased exercise and wellness program for people diagnosed with Parkinson disease: Experiences from a 10-month trial. J Geriatr Phys Ther, 35, 173-180.

[12] Oguh O, Eisenstein A, Kwasny M, \& Simuni T (2014) Back to the basics: Regular exercise matters in Parkinson's disease: Results from the National Parkinson Foundation QII Registry study. Parkinsonism Relat Disord, 20, 1221-1225.

[13] Factor SA, Bennett A, Hohler AD, Wang D, \& Miyasaki JM (2016) Quality improvement in neurology: Parkinson disease update quality measurement set: Executive summary. Neurology, 86, 2278-2283.

[14] Pate RR, Yancey AK, \& Kraus WE (2010) The 2008 Physical Activity Guidelines for Americans: Implications for clinical and public health practice. Am J Lifestyle Med, 4, 9.

[15] Okun MS, Siderowf A, Nutt JG, O'Conner GT, Bloem BR, Olmstead EM, Guttman M, Simuni T, Cheng E, Cohen EV, Parashos S, Marsh L, Malaty IA, Giladi N, Schmidt P, \& Oberdorf J (2010) Piloting the NPF data-driven quality improvement initiative. Parkinsonism Relat Disord, 16, 517-521.

[16] Nutt JG, Siderowf AD, Guttman M, Schmidt PN, Zamudio JI, Wu SS, Okun MS, Simuni T, Parashos SA, Dahodwala NA, Davis TL, Giladi N, Gurevich T, Hauser RA, Jankovic J, Lyons KE, Marsh L, Miyasaki JM, Morgan JC, Santiago AJ, Tarsy D, Mari Z, Malaty IA, Nelson EC, \&
National Parkinson Foundation Quality Improvement Initiative Investigators (2014) Mobility, mood and site of care impact health related quality of life in Parkinson's disease. Parkinsonism Relat Disord, 20, 274-279.

[17] Hagell P, \& Nygren C (2007) The 39 item Parkinson's disease questionnaire (PDQ-39) revisited: Implications for evidence based medicine. J Neurol Neurosurg Psychiatry, 78, 1191-1198.

[18] Podsiadlo D, \& Richardson S (1991) The timed "Up \& Go": A test of basic functional mobility for frail elderly persons. J Am Geriatr Soc, 39, 142-148.

[19] Peto V, Jenkinson C, \& Fitzpatrick R (2001) Determining minimally important differences for the PDQ-39 Parkinson's disease questionnaire. Age Ageing, 30, 299-302.

[20] Fitzpatrick R, Norquist JM, \& Jenkinson C (2004) Distribution-based criteria for change in health-related quality of life in Parkinson's disease. J Clin Epidemiol, 57, 40-44.

[21] Huang SL, Hsieh CL, Wu RM, Tai CH, Lin CH, \& Lu WS (2011) Minimal detectable change of the timed "up \& go" test and the dynamic gait index in people with Parkinson disease. Phys Ther, 91, 114-121.

[22] Dal Bello-Haas V, Klassen L, Sheppard MS, \& Metcalfe A (2011) Psychometric properties of activity, self-efficacy, and quality-of-life measures in individuals with Parkinson disease. Physiother Can, 63, 47-57.

[23] Steffen T, \& Seney M (2008) Test-retest reliability and minimal detectable change on balance and ambulation tests, the 36-item short-form health survey, and the unified Parkinson disease rating scale in people with parkinsonism. Phys Ther, 88, 733-746.

[24] Hackney ME, \& Earhart GM (2010) Effects of dance on balance and gait in severe Parkinson disease: A case study. Disabil Rehabil, 32, 679-684.

[25] Stegemoller EL, Schmidt P, Hass C, Malaty I, Okun MS, \& National Parkinson Foundation Quality Improvement Initiative Investigators (2015) How should pushing off or the use of assistive devices be incorporated in the timed up and go for persons with Parkinson disease? Arch Phys Med Rehabil, 96, $1728-1732$

[26] Del Din S, Godfrey A, Mazza C, Lord S, \& Rochester L (2016) Free-living monitoring of Parkinson's disease: Lessons from the field. Mov Disord, 31, 1293-1313.

[27] Ball TJ, Joy EA, Gren LH, \& Shaw JM (2016) Concurrent validity of a self-reported physical activity "vital sign" questionnaire with adult primary care patients. Prev Chronic Dis, 13, E16.

[28] Helmerhorst HJ, Brage S, Warren J, Besson H, \& Ekelund U (2012) A systematic review of reliability and objective criterion-related validity of physical activity questionnaires. Int J Behav Nutr Phys Act, 9, 103. 


\section{APPENDIX}

Current NPF-QII (Parkinson's Outcomes Project) Investigators: site principal investigators and current participating centers

1. Bastiaan Bloem, MD: Nijmegen Parkinson Center, Nijmegen, Netherlands

2. Nabila Dahodwala, MD: University of Pennsylvania, Pennsylvania Hospital, Philadelphia, PA, USA

3. Thomas Davis, MD: Vanderbilt University Medical Center, Nashville, TN, USA

4. Rohit Dhall, MD: Parkinson's Institute and Clinical Center, Sunnyvale, CA, USA

5. Tanya Gurevich, MD: Tel Aviv Sourasky Medical Center, Tel Aviv, Israel

6. Mark Guttman, MD: Centre for Movement Disorders, Markham Stouffville Hospital, Toronto, Canada

7. Robert Hauser, MD, MBA: University of South Florida Parkinson's Disease \& Movement Disorders Center, Tampa, FL, USA

8. Joseph Jankovic, MD: Baylor College of Medicine, Houston, TX, USA

9. Kelly Lyons, PhD: University of Kansas Medical Center, Kansas City, KS, USA
10. Irene Malaty, MD: University of Florida Parkinson's Disease and Movement Disorders Center, Gainesville, FL, USA

11. Zoltan Mari, MD: Johns Hopkins Parkinson's Disease and Movement Disorders Center, Baltimore, MD, USA

12. Connie Marras, MD: Toronto Western Hospital Movement Disorders Center, Toronto, Canada

13. John Morgan, MD: Georgia Health and Sciences University, Augusta, GA, USA

14. Fernando Pagan, MD: Georgetown University Hospital, Washington, DC, USA

15. Sotirios Parashos, MD: Struthers Parkinson's Center, Golden Valley, MN, USA

16. Joe Quinn, MD: Oregon Health \& Science University Parkinson Center, Portland, OR, USA

17. Naomi Salins, MD: Muhammad Ali Parkinson Center, Phoenix, AZ, USA

18. David Simon, MD: Beth Israel Deaconess Medical Center, Boston, MA, USA

19. Tanya Simuni, MD: Northwestern University Parkinson's Disease \& Movement Disorders Center, Chicago, IL, USA

20. Carlos Singer, MD: University of Miami, Miami, FL, USA 